Article

\title{
Multitask Saliency Detection model for SAR Image and Its Application in SAR and Optical Image Fusion
}

\author{
Chunhui Liu ${ }^{1,2, *}$, Yue Qi ${ }^{2}$ and Wenrui Ding ${ }^{1,3}$ \\ 1 Unmanned Systems Research Institute, Beihang University, Beijing 100191, China; \\ ding@buaa.edu.cn (W. D.) \\ 2 State Key Laboratory of Virtual Reality Technology and System, Beihang University, Beijing 100191, China; \\ qy@buaa.edu.cn (Y.Q.) \\ 3 Collaborative Innovation Centre of Geospatial Technology, Wuhan 430000, China \\ * Correspondence: liuchunhui2134@126.com; Tel.: +86-10-8231-7397
}

\begin{abstract}
Saliency detection in synthetic aperture radar (SAR) image is a difficult problem. This paper proposed a multitask saliency detection (MSD) model for the saliency detection task of SAR image. Firstly, we extract four features of SAR image as the input of the MSD model, which include the intensity, orientation, uniqueness and global contrast. Then, the saliency map is generated by the multitask sparsity pursuit (MTSP) which integrates the multiple features collaboratively. Subjective and objective evaluation of the MSD model verifies its effectiveness. Based on the saliency maps of the source images, an image fusion method is proposed for the SAR and color optical image fusion. The experimental results of real data show the proposed image fusion method is superior to the presenting methods in terms of several universal quality evaluation indexes, as well as in the visual quality. The salient areas in the SAR image can be highlighted and the spatial and spectral details of color optical image can also be preserved in the fusion result.
\end{abstract}

Keywords: synthetic aperture radar; features extraction; saliency detection; image fusion

\section{Introduction}

\subsection{Saliency detection of SAR image}

Synthetic aperture radar (SAR) technology is one of the most important advances in the field of remote sensing in recent decades. Among the broad applications of SAR, automatic target recognition (ATR) is hot issue. Conventional target detection algorithms include constant false alarm rate (CFAR) or support vector machines (SVM), etc, where the detector is applied in pixel-by-pixel manner. In recent years, visual saliency detection has been of great research interest. Although many saliency detection models have been proposed in the past few years, they mainly focus on the natural images [1-3] rather than SAR images. Because the SAR images are very different from the visible images in terms of the gray scale feature, some objects such as flat roads, airports and bridges, are bright in natural images but dark in SAR images. This difference makes the classical saliency detection method for natural images not applicable to SAR images, even these methods completely failed in some cases. Figure 1 shows a failure case of the saliency detection for SAR image by the classical Itti's method [1]. Figure 1(a) is a color image of a runway, and Figure 1(b) is the corresponding SAR image of Figure 1(a) after registration. Figure 1(c) shows the saliency map detected by Itti's method. As shown in Figure 1(c), the scene surrounding the runway is "highlighted", but the runway itself and the corner reflectors on the runway cannot be detected by Itti's method. This is because the traditional salieny detection models designed for the SAR images have not taken into account the inherent characteristics of the SAR images, such as intensity, direction and point target, etc. So it is essential to establish saliency detection model designed specifically for the SAR images. 


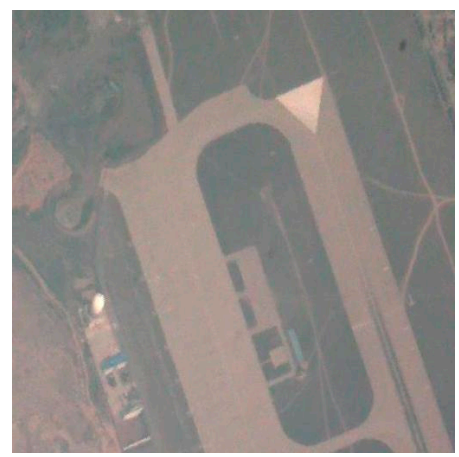

(a) natural image

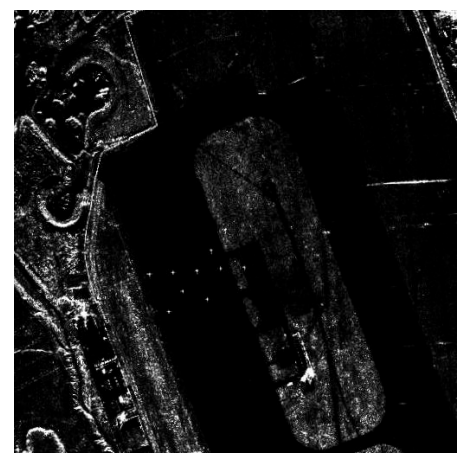

(b) SAR image

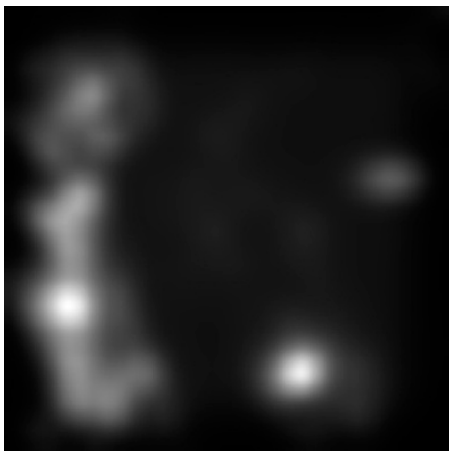

(c) Result by Itti's model

Figure 1. Failure case of saliency detection of SAR image

Until now, limited number of work has tried to apply saliency detection to SAR images. Amoon et al. [4] introduced it in SAR image ship detection, their method can remove disturbs come from the land. Bo et al. [5] proposed a low-level visual feature based SAR image saliency detection method, which performs well for detecting small objects. Jäger et al. [6] considered the saliency of SAR images from the perspective of entropy and change in the context, while Zhang et al. [7] proposed a self-adaptive neighborhood based SAR image de-speckling method by combining the saliency map and threshold selection.

In the recent research, most scholars start to combine the visual attention model with other methods to enhance the saliency detection result. Dou [8] combines the strength of gradient saliency map and scattering structure features to improve accuracy and efficiency of target detection in SAR images. Wang [9] proposed a new hierarchical saliency filtering method for fast and accurate ship detection in high-resolution SAR images. Song [10] proposed a region- and edge-based convex active contour model, which combines the advantages of a multi-scale saliency detection method to address a target detection problem in a large-scale and high-resolution SAR image automatically.

Generally most methods in the field of saliency detection of SAR images draw on the human visual attention mechanism which is based on the "bottom-up" visual attention framework [1,4-8]. The "bottom-up" mechanism requires that the features of SAR images can be analyzed and extracted firstly. However, the current saliency detection models have not fully analyzed the features of the SAR images. Meanwhile, the cross-feature information has not been well considered, thus a more accurate saliency detection result cannot be obtained by the existing methods.

\subsection{SAR and optical image fusion}

With the rapid developments in the field of aviation technology, many novel platform, such as unmanned aerial vehicle (UAV), start to play more and more important role in the remote sensing application. The modern airborne surveillance and reconnaissance systems are usually multi-sensor system, which is always consisted of optical camera, infrared camera (IF), synthetic aperture radar (SAR), etc. Due to the all-weather and all-time ability, SAR imaging system can provide much complementary information to optical images. SAR and optical image fusion can combine information from each source images of the same scene into one single image to obtain data that is more useful than that from the individual source image. It also can reduce imprecision and uncertainty in the spatial properties and maintain completeness of the spectral information.

For the SAR imaging system, we mainly focus on the areas where more salient target may exsit in SAR images. For the optical and SAR image fusion, the more salient the area in the SAR image is, the higher proportion of the area should be integrated into the fusion result. The areas where ambient or less salient object exists have no necessity to be fused into the image. Thus, introducing the saliency information of the source images into the fusion procedure can hopefully enhance the fusion performance in SAR and optical image fusion.

Various multi-sensor image fusion methods have been developed. Basically, the existing fusion methods can be categorized into two categories. One category is the spatial domain based methods, which directly fuse the source images in the pixel level. The representative spatial domain-based 
methods are the intensity-hue-saturation (IHS) and its variants [11,12], and the principal component analysis (PCA) [13], etc. The other category is the transformed domain based methods, which achieve the fusion procedure with certain frequency or time-frequency transforms. The most popular transform domain based methods are based on the multi-scale transforms. The commonly used multi-scale transforms include various pyramids [14], discrete wavelet transform (DWT) [15], contourlet [16], etc. Recently, sparse representation (SR) and its variants [17-20] have produced state-of-the-art results in multi-sensor image fusion, which exploit the self-similarity properties of natural images.

However, most of the existing image fusion methods are less use of the salient information. Although some of the methods based on multi-resolution analysis (MRA) [21] have used the saliency information to develop the fusion strategy, they mainly depend on the "regional segment" of the source image based on the saliency map. This lead to the fact that obvious "block effect" appears on the fusion result, and the relationship between saliency information and the fusion procedure is separated. Therefore, an effective integration of the saliency information and the image fusion need to be explored in SAR and optical image fusion.

\subsection{Our work}

In this paper, we present a multitask saliency detection (MSD) model based on multitask sparse pursuit (MTSP) [22] which treat saliency detection as a sparsity pursuit problem and integrate multiple types of features for detecting saliency collaboratively. Before that, the four features of the SAR image are extracted which include the intensity, orientation, uniqueness and global contrast. All these feature maps are constructed and normalized as the input of the MTSP model, which can integrate multiple visual features and cross-feature information into a unified inference procedure. Then a more accurate and reliable saliency detection result can be provided for the further application of the saliency map.

Based on the saliency information of the SAR image obtained by MSD model, we develop a fully automatic SAR and optical image fusion method with saliency preserving. Firstly, we build up the fusion gradient field for the source images based on structure tensor. Then, a normalized weight is assigned to each pixel in the source images according to the saliency maps of the source images. At last, the variational optimization problem is solved to obtain the fusion result closest to the source image in the sense of the weighted gradient. We test this fusion procedure on the application of SAR and color optical image fusion. The experimental results show that our method allows the spatial and spectral details of optical image data to be preserved and to better characterize the salient areas in the source images.

In Section 2, the proposed MSD model is described in detail. The procedure of SAR and optical image fusion is introduced in Section 3. In Section 4, we compare the saliency detection and fusion results obtained by our method with that of existing methods. Discussion and conclusions are presented in Section 5 and Section 6, respectively.

\section{Multi-task Saliency Detection Model for SAR image}

\subsection{Model overview}

Our proposed saliency detection model-MSD model refers to the human visual mechanism to explore the saliency detection of SAR image. We expect to select the features that reflected the characteristics of the SAR image as much as possible. Based on the analysis of the characteristics of the SAR images, we select four features including intensity, orientation, uniqueness and global contrast. The intensity feature characterizes the difference between the target and the background, the orientation feature characterizes the azimuth of the target in different directions, the uniqueness feature highlights the obvious target (such as point target) and the global contrast feature characterizes the global prominence of the potential target region. These features are adopted as the input of the multitask sparse pursuit-MTSP. The saliency map is produced by the output of MTSP. Figure 1 presents the workflow of the proposed MSD model. 


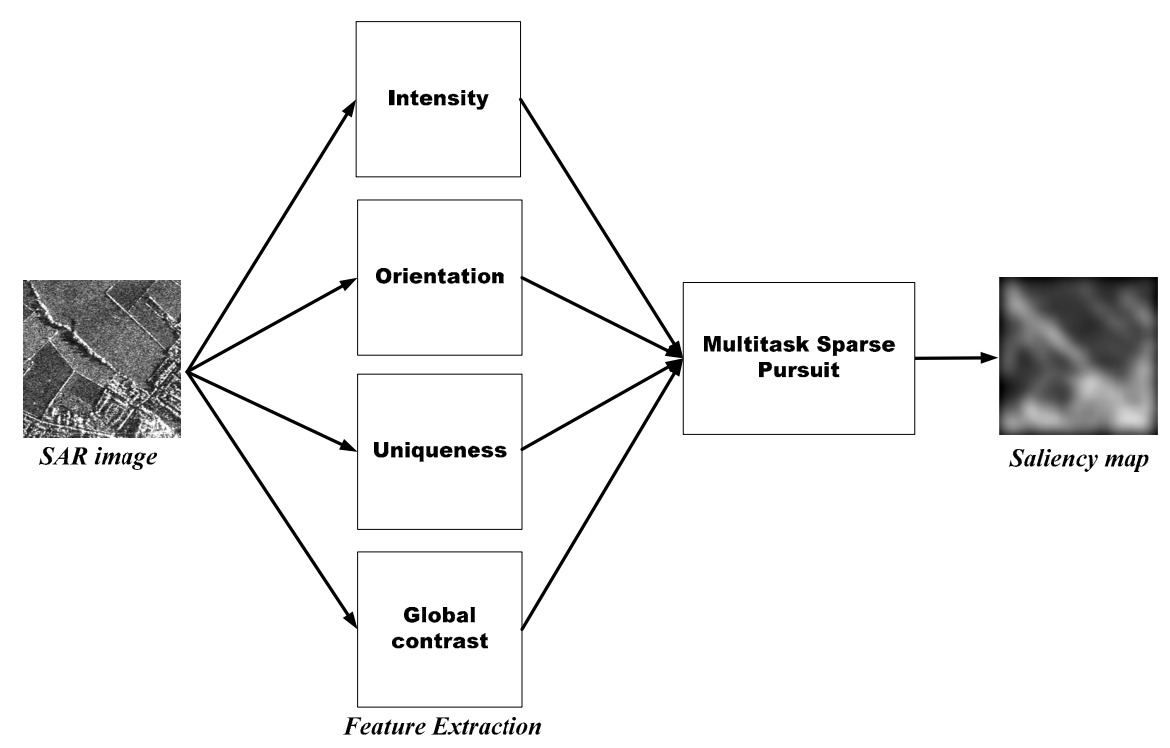

Figure 2. Workflow of the Multitask Saliency Detection Model

\subsection{Features extraction}

As the basis of the proposed saliency detection model, how to extract the four typical features of the SAR image is a key issue. The mathematical expression and its corresponding calculation method of these features is introduced as follow.

\section{(1) Intensity feature}

The intensity feature is firstly produced by the gray level co-occurrence matrix (GLCM) [21] which describes how often on gray level appears in a specified spatial relationship with another gray level. The entry at $(i, j)$ of the GLCM indicates the number of occurrences of the pair of gray. After construction of GLCM, we choose the entropy measure of GLCM intensity descriptor. Entropy measures the randomness of the elements of the co-occurrence matrix. It is maximum when elements in the matrix are equal, while is equal to 0 if all elements are different.

$$
\begin{aligned}
& \text { Entropy }=-\sum_{i}^{K} \sum_{i}^{K} p_{i j} \log _{2}\left(p_{i j}\right) \\
& C_{\text {intensity }}=N(\text { Entropy })
\end{aligned}
$$

where $p_{i j}$ represents the elements of the co-occurrence matrix and $K$ is the number of gray levels used to reduce the size of the matrix, which is set 16 in our experiment, $N(\bullet)$ is the normalization operator and $C_{\text {intensity }}$ is the intensity feature map.

\section{(2) Orientation feature}

The orientation feature is obtained from the source images using 2D Gabor filters [23], which are the product of a cosine grating and a 2D Gaussian envelope and can be expressed as follow:

$$
H\left(x, y, \theta_{k}, \lambda, \alpha, \beta\right)=\frac{1}{2 \pi \alpha \beta} \exp \left\{-\pi\left[\left(\frac{x_{\theta_{i}}}{\alpha}\right)^{2}+\left(\frac{y_{\theta_{i}}}{\beta}\right)^{2}\right]\right\} \exp \frac{2 \pi i x_{\theta_{i}}}{\lambda}
$$

where $\alpha$ and $\beta$ are the standard deviation on the $\mathrm{X}$ axis and $\mathrm{Y}$ axis of the Gaussian function respectively, $\theta_{k}$ and $\lambda$ denotes the phase and wavelength of Sine wave. $\theta_{k}, x_{\theta_{k}}$ and $y_{\theta_{k}}$ are calculated by the following equations: 


$$
\begin{aligned}
& \theta_{k}=\frac{\pi}{n}(k-1), k=1,2, \ldots n \\
& x_{\theta_{k}}=x \cos \left(\theta_{k}\right)+y \sin \left(\theta_{k}\right) \\
& y_{\theta_{k}}=-x \sin \left(\theta_{k}\right)+y \cos \left(\theta_{k}\right)
\end{aligned}
$$

When $\theta_{k}$ is selected as different value, the Gabor filters on $n$ different directions can be obtained. We set $n=4$ to get four orientation feature maps on the orientations of $0^{\circ}, 45^{\circ}, 90^{\circ}$ and $135^{\circ}$. The final orientation feature map can be obtained by the linear sum of the four feature maps $F_{\text {Ori }}(\theta)$, as follow:

$$
C_{\text {Orientation }}=\underset{\theta \in\left\{0^{\circ}, 45^{\circ}, 90^{\circ}, 135^{\circ}\right\}}{\oplus} N\left(F_{\text {Ori }}(\theta)\right)
$$

where $C_{\text {Orientation }}$ denotes the final orientation feature map, $\oplus$ denotes the linear addition operation on the corresponding pixels of the four feature maps .

\section{(3) Uniqueness feature}

According to the characteristics of SAR imaging system, some targets such as the corner reflector object formed as the point targets which are very sparse in the SAR images. We believe the point targets are very unique, so we can use pixel frequency to represent the uniqueness feature of the image. We compute the $98^{\text {th }}$ percentile of the SAR image which is present as $Z_{98}$. In a search region of $3 \times 3$,if more than 6 pixels have greater values than $Z_{98}$, all of the pixels in the region will be retained, otherwise the region will be filled with black. Through normalized operation, the uniqueness feature map $C_{t}$ can be obtained.

\section{(4) Global contrast feature}

Global contrast feature can be defined as the difference between a region (pixel) and the whole image. For the pixel $I(x, y)$, its global contrast feature map can be quantified as follow:

$$
\begin{aligned}
& \operatorname{val}(x, y)=I(x, y)-\frac{1}{N \times M} \sum_{i \in N} \sum_{j \in M} I(x, y) \\
& g(x, y)=\left\{\begin{array}{l}
\operatorname{val}(x, y), \operatorname{val}(x, y) \geq \alpha \\
0 \quad, \operatorname{val}(x, y)<\alpha
\end{array}\right. \\
& C_{\text {Global contrast }}=N(g(x, y))
\end{aligned}
$$

where $g(x, y)$ denotes the global contrast at location $(x, y), \alpha$ is a parameter that can be adjusted artificially (we set it as the mean value of the entire image pixel ) and $C_{\text {Global contrast }}$ is the final global contrast feature map.

\subsection{Generation of saliency map}

The MTSP method [22] treats the saliency detection as a sparsity pursuit problem and integrates multiple types of features for detecting saliency collaboratively. Since the cross-feature information has been well considered, such a joint inference scheme can produce more accurate and reliable results than the models of producing the saliency maps individually.

The MTSP can be seen as a multitask generalization of the low-rank representation (LRR). MTSP seeks a jointly sparse matrix $E$ by solving the following convex optimization problem:

$$
\begin{aligned}
& \min _{\substack{Z_{1}, \ldots, Z_{K} \\
E_{1}, \ldots, \mathrm{E}_{K}}} \sum_{i=1}^{K}\left\|Z_{i}\right\|_{*}+\lambda\|E\|_{2,1} \\
& \text { s.t. } X_{i}=X_{i} Z_{i}+E_{i}, i=1, \ldots K
\end{aligned}
$$

where $\|\bullet\|_{*}$ denotes the matrix nuclear norm, parameter $\lambda>0$ is balance factor and $\|\bullet\|_{2,1}$ is the $\ell_{2,1}$ norm defined as the sum of $\ell_{2}$ norms of the columns of a matrix, as follow: 


$$
\|E\|_{2,1}=\sum_{i} \sqrt{\sum_{j}(E(j, i))^{2}}
$$

where $E(j, i)$ is the $(j, i)$ th entry of $E$. And $X_{i}$ denotes the $i$ th type of feature.

In Eq.(6), $E_{i}$ is the $i$ th saliency feature (here $\left.K=4\right)$ and they are $C_{\text {intensity }}, C_{\text {Orientation }}, C_{\text {Uniqueness }}$ and $C_{\text {global contrast }}$ for SAR image. $E=\left[E_{1} ; E_{2} ; \cdots ; E_{K}\right]$ denotes the joint matrix by vertically concatenating $E_{1}, E_{2}, \cdots E_{K}$ together along the column. The non-salient and salient targets are identified by $X_{i} Z_{i}$ and $Z_{i}$, respectively. Since the columns in different matrices with the same index correspond to the same image patch, this technique is also to encourage different features to produce consistent saliency maps. In this way, the cross-feature information is naturally modeled such that the multiple features will take effects collaboratively.

Problem (5) is convex and can be optimized, and it can be converted into the following equivalent problem:

$$
\begin{aligned}
& \min _{\substack{J_{1}, \ldots, J_{K} \\
Z_{1}, \ldots, Z_{K} \\
E_{1}, \ldots, \mathrm{E}_{K}}} \sum_{i=1}^{K}\left\|J_{i}\right\|_{*}+\lambda\|E\|_{2,1} \\
& \text { s.t. } X_{i}=X_{i} Z_{i}+E_{i} \\
& \quad Z_{i}=J_{i}, i=1, \ldots, K .
\end{aligned}
$$

This problem can be solved with the ALM method [22], and the optimization procedure is listed as follow:

Input: Data matrices $\left\{X_{i}\right\}$ and parameter $\lambda$ (we set $\lambda=0.05$ in our implementation).

While not converged do

Step 1: Fix the others and update $J_{1}, J_{2}, \cdots J_{K}$ by

$J_{i}=\arg \min _{J} \frac{1}{\mu}\|J\|_{*}+\frac{1}{2}\left\|J_{i}-\left(Z_{i}+\frac{W_{i}}{\mu}\right)\right\|_{F}^{2}$

Step 2: Fix the others and update $Z_{1}, Z_{2}, \cdots Z_{K}$ by

$Z_{i}=M\left(X_{i}^{T}\left(X_{i}-E_{i}\right)+J_{i}+\frac{X_{i}^{T} Y_{i}-W_{i}}{\mu}\right)$

where $M=\left(I+\sum_{i=1}^{K} X_{i}^{T} X_{i}\right)^{-1}$

Step 3: Fix the others and update $E=\left[E_{1} ; E_{2} ; \cdots ; E_{K}\right]$ by

$E=\arg \min _{E} \frac{\lambda}{\mu}\|E\|_{2,1}+\frac{1}{2}\|E-G\|_{F}^{2}$

where $G$ is formed by vertically concatenating the matrices $X_{i}-X_{i} Z_{i}+Y_{i} / \mu, i=1, \cdots, K$ together along column.

Step 4: Update the multipliers

$$
\begin{aligned}
& Y_{i}=Y_{i}+\mu\left(X_{i}-X_{i} Z_{i}-E_{i}\right) \\
& W_{i}=W_{i}+\mu\left(Z_{i}-J_{i}\right)
\end{aligned}
$$

Step 5: Update the parameter $\mu$ by

$\mu=\min \left(\rho \mu, 10^{10}\right)$

where the parameter $\rho$ decides the convergence speed. It is set as $\rho=1.1$ in our experiments.

Step 6: Check the convergence condition: $X_{i}-X_{i} Z_{i}-E_{i} \rightarrow 0$ and $Z_{i}-J_{i} \rightarrow 0, i=1, \ldots, K$.

end while

Output: The optimal solution $E^{*}$. 
Let $E^{*}=\left\{E_{1}^{*}, \cdots, E_{K}^{*}\right\}$ be the optimal solution to problem (5). We quantify the response of the sparse matrices as follow to obtain a saliency value for $i$ th patch $P_{i}$ :

$$
S\left(P_{i}\right)=\sum_{j=1}^{K}\left\|E_{j}^{*}(:, i)\right\|_{2}
$$

where $\left\|E_{j}^{*}(:, i)\right\|_{2}$ denotes the $\ell_{2}$ norm of the $i$ th column of $E_{j}^{*}$. The final saliency map is synthesized by the saliency values of each patch (from Equation (9)).

\section{SAR and optical image fusion guided by saliency map}

For the SAR and color optical image fusion application, we design the practical fusion procedure which integrates the saliency information of the source images, as shown in Figure 3.

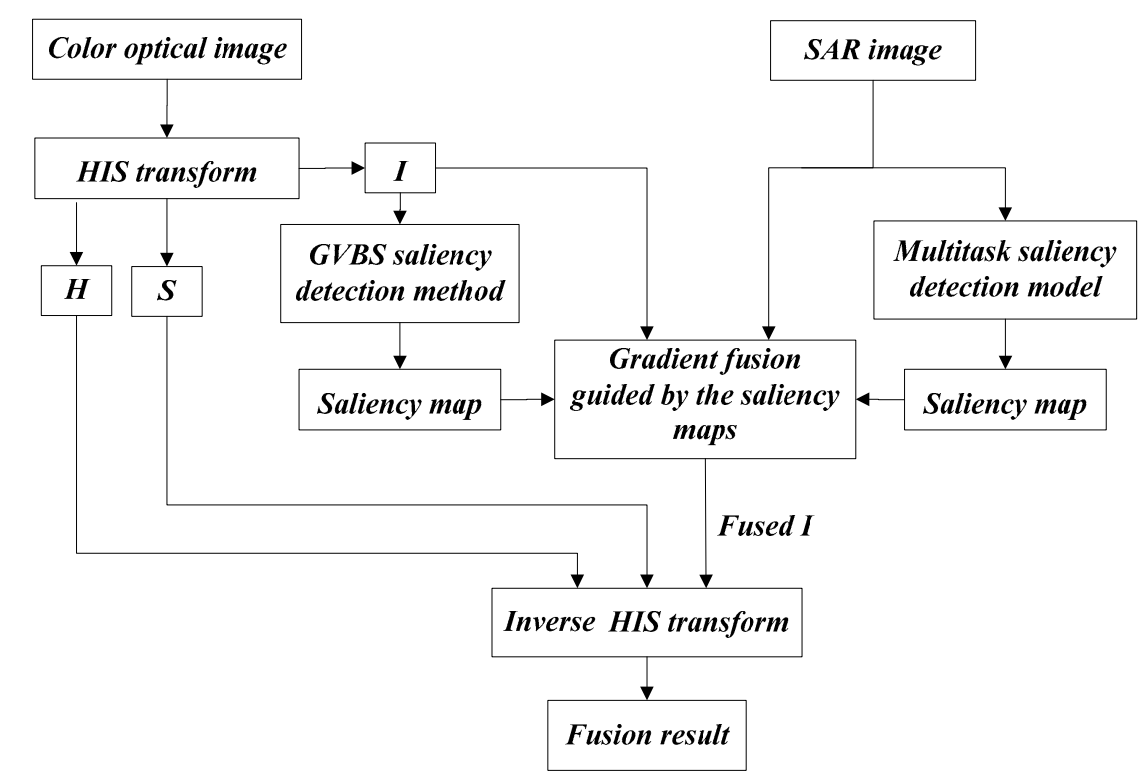

Figure 3. Fusion procedure of the SAR and color optical image

Firstly, the color optical image is firstly transformed from RGB color space to IHS color space by IHS transform as following Equation:

$$
\begin{aligned}
& \left(\begin{array}{l}
I \\
v_{1} \\
v_{2}
\end{array}\right)=\left(\begin{array}{ccc}
1 / \sqrt{3} & 1 / \sqrt{3} & 1 / \sqrt{3} \\
1 / \sqrt{6} & 1 / \sqrt{6} & -2 / \sqrt{6} \\
1 / \sqrt{2} & 1 / \sqrt{2} & 0
\end{array}\right)\left(\begin{array}{l}
R \\
G \\
B
\end{array}\right) \\
& H=\tan ^{-1}\left(v_{2} / v_{1}\right) \\
& S=\sqrt{v_{1}^{2}+v_{2}^{2}}
\end{aligned}
$$

where $I$ denotes intensity, $H$ denotes hue and $S$ denotes saturation. The hue component $H$ and saturation component $S$ are preserved for the inverse IHS transform in the final step, and the intensity component $I$ is used to fuse with SAR image.

We adopt the variational model [24] to perform the image fusion progress, which can preserve the salient information and enhance the contrast for visualization. The saliency map of SAR image is obtained by the proposed MSD model described above, which is normalized as $S_{1}$. The saliency map of optical image is obtained by a fast and efficient saliency detection model-GBVS method [25], which is normalized as $S_{2}$. The saliency maps $S_{1}$ and $S_{2}$ are adopted to guide the fusion progress which critical point is to construct the target gradient $V$ which should reflect the salient features of the source image as much as possible.

To better balance the weight of each source image in the fused image, we proposed the normalized weight factor for each source image as follow: 


$$
\begin{aligned}
& \mathrm{w}_{1}(x, y)=\frac{S_{1}(x, y)}{\sqrt{S_{1}(x, y)^{2}+S_{2}(x, y)^{2}}} \\
& \mathrm{w}_{2}(x, y)=\frac{S_{2}(x, y)}{\sqrt{S_{1}(x, y)^{2}+S_{2}(x, y)^{2}}}
\end{aligned}
$$

We construct the weighted structure tensor [26] as follow:

$$
C(x, y)=\left[\begin{array}{cc}
\sum_{k}\left(\mathrm{w}_{k} \frac{\partial I_{k}}{\partial x}\right)^{2} & \sum_{k} \mathrm{w}_{k}^{2} \frac{\partial I_{k}}{\partial x} \cdot \frac{\partial I_{k}}{\partial y} \\
\sum_{k} \mathrm{w}_{k}^{2} \frac{\partial I_{k}}{\partial x} \cdot \frac{\partial I_{k}}{\partial y} & \sum_{k}\left(\mathrm{w}_{k} \frac{\partial I_{k}}{\partial y}\right)^{2}
\end{array}\right]
$$

where $C(x, y)$ denotes the weighted structure tensor and $I_{k}$ denotes the $k$ th source image.

The target gradient $V(x, y)$ at pixel $(x, y)$ is constructed by eigen-decomposition on $C(x, y)$. The square root of the maximum eigen-value of $C(x, y)$ is the magnitude of gradient and corresponding eigen-vector is the direction respectively. To make the orientation of $V$ to agree with that of the gradient of the average of all inputs, the following expression of the target gradient is presented:

$$
V(x, y)=\sqrt{\lambda+} \cdot \theta^{+} \cdot \operatorname{sign}\left(\theta^{+} \cdot \sum_{k} \mathrm{w}_{k}(x, y) \nabla I_{k}(x, y)\right)
$$

where $\lambda+$ is the maximum eigenvalue of $C(x, y)$ and $\theta^{+}$is the corresponding eigenvector.

The fusion result $I_{f}(x, y)$ can be found by minimizing the following optimization problem [27]:

$$
\begin{aligned}
& \min \iint_{\Omega}\left|\nabla I_{f}(x, y)-V(x, y)\right|^{2} d x d y \\
& \text { s.t. } I_{f}(x, y) \in[0,255]
\end{aligned}
$$

Such a function as Equation (14) can be solved by the iterative steps as follow:

$$
\left\{\begin{array}{l}
I_{f}(x, y)^{t+1 / 2}=I_{f}(x, y)^{t}+1 / 4\left(\Delta I_{f}(x, y)^{t}-\operatorname{div} V(x, y)\right) \\
I_{f}(x, y)^{t}=\max \left(0, \min \left(255, I_{f}(x, y)^{t+1 / 2}\right)\right)
\end{array}\right.
$$

Based on the equations above, the fused intensity $I_{f}$ can be obtained. Finally, the fusion result is obtained by performing inverse IHS transform of the original $H$ channel, $S$ channel of the color optical image and new $I$ channel-the fused intensity $I_{f}$. The final fusion result in RGB color space could be calculated by following equations:

$$
\begin{aligned}
& v_{1}=S \cdot \cos (H) \\
& v_{2}=S \cdot \sin (H) \\
& \left(\begin{array}{l}
R \\
G \\
B
\end{array}\right)=\left(\begin{array}{ccc}
1 / \sqrt{3} & 1 / \sqrt{6} & 1 / \sqrt{2} \\
1 / \sqrt{3} & 1 / \sqrt{6} & -/ \sqrt{2} \\
1 / \sqrt{3} & -2 / \sqrt{6} & 0
\end{array}\right)\left(\begin{array}{l}
I_{f} \\
v_{1} \\
v_{2}
\end{array}\right)
\end{aligned}
$$

\section{Experimental Results}

\subsection{Study region and dataset}

This section presents the experimental results. We use a dataset of airborne SAR and color optical image which is taken by a medium-altitude unmanned aerial vehicle (UAV) that cruises at an 
altitude of $3000 \mathrm{~m}$. A SAR imaging system and a CCD camera are mounted together on the UAV, which formed a multi-sensor system. The study region locates in an urban area in central plains of China. Figure 4 shows the study region and corresponding flight course.

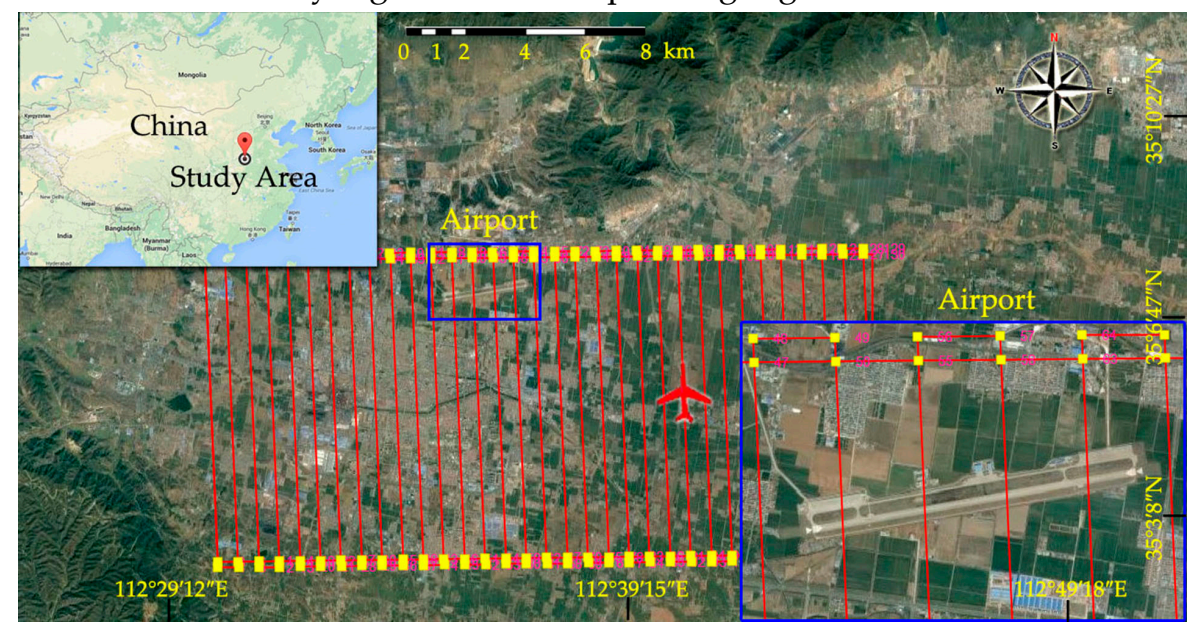

Figure 4. Study region and flight course.

Two sets of co-registered SAR and color images are used to evaluate the proposed mode, which are shown in Figure 5.

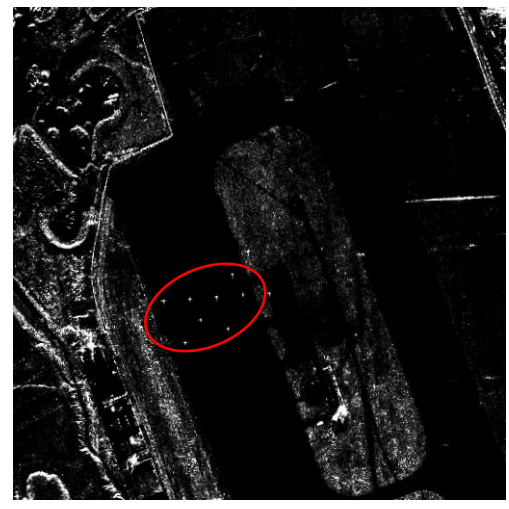

(a) SAR image of "Airport"

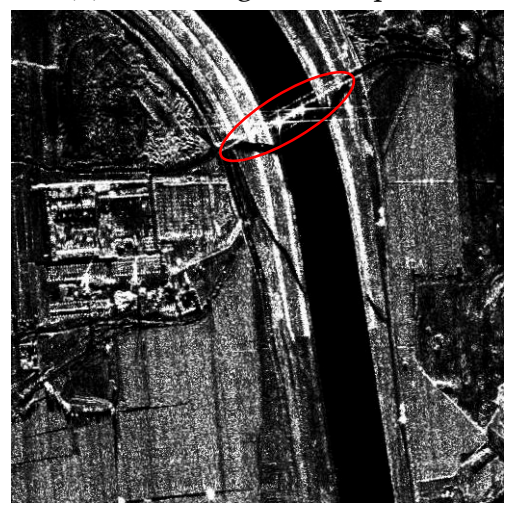

(c) SAR image of "Bridge"

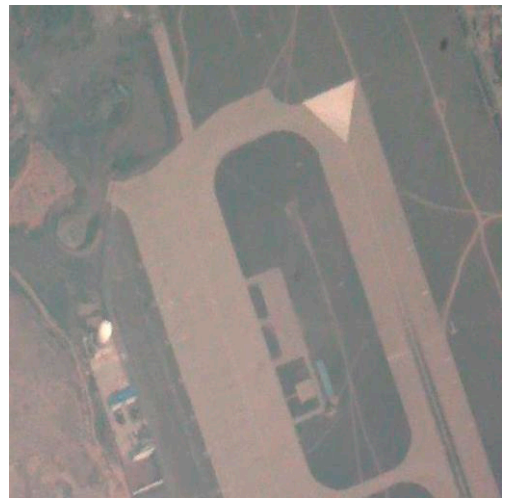

(b) Optical image of "Airport"

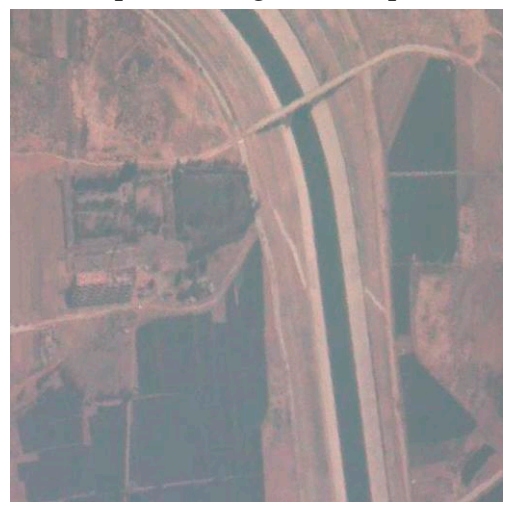

(d) Optical image of "Bridge"

Figure 5. Experimental dataset.

Figure 5(a) and Figure 5(b) show the SAR and optical images of "Airport". Inside the red circle of Figure 5(a), there are several corner reflectors made of metal used to test the SAR imaging system, while they cannot be seen in the optical image of Figure 5(b). The other group of data, Figure 5(c) and Figure 5(d), show the SAR and optical images of "Bridge". We can see there are strong backscattering effects on the bridge which may come from the vehicles riding over the bridge. However, due to the high scene depth, we can hardly see the any moving objects in Figure 5(d). In general, the optical images show better spectral quality but with little mist, while the SAR image is not sensitive to the mist and has a good reflective character to metal. 
We validated the proposed MSD model on the saliency detection of SAR images. After that, the SAR and optical image fusion by the proposed fusion procedure in Figure 3 is also tested.

\subsection{Validation on saliency detection of SAR images}

The proposed MSD model is compared with four existing saliency detection methods, which are selected as benchmarks, i.e., the CFAR [28] approach and three saliency-based approaches, namely SoP [29], PCT [30], and PR [31]. CFAR is the conventional method of target detection in SAR images. A threshold is calculated by the false alarm rate of statistical distribution and applied to the image to obtain the detection result. However, if a target in the SAR image has similar intensity level as its surroundings, CFAR leads to high false-alarm rate. SoP is a SAR image saliency detection algorithm that considers the local, global saliency information and the statistical distribution of SAR images. PCT is a bottom-up attention model based on pulsed Hebbian neural networks, which is directly applied to ship detection in SAR images. PR is a new approach for saliency detection of SAR image based on the idea of pattern recurrence which quantifies the saliency of a local patch by how well it can be reconstructed from patches in the background.

Figure 6 and Figure 7 show the saliency detection results of CFAR, SoP, PCT, RP and the proposed method applied to the SAR images in Figure 5(a) and Figure 5(c). The corresponding original SAR image is also given in Figure 6 and Figure 7 as reference.

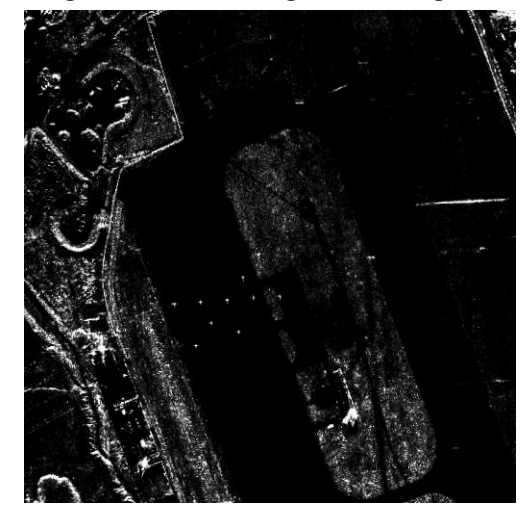

(a) original SAR image of "Airport"

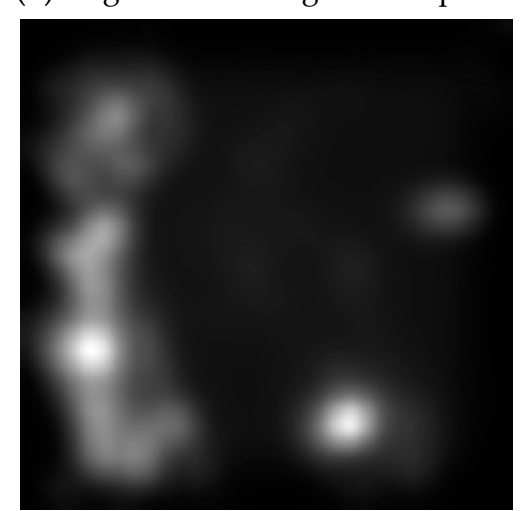

(d) result of PCT method

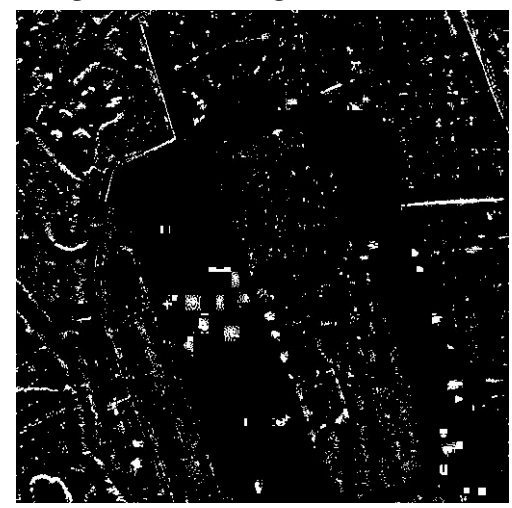

(b) result of CFAR method

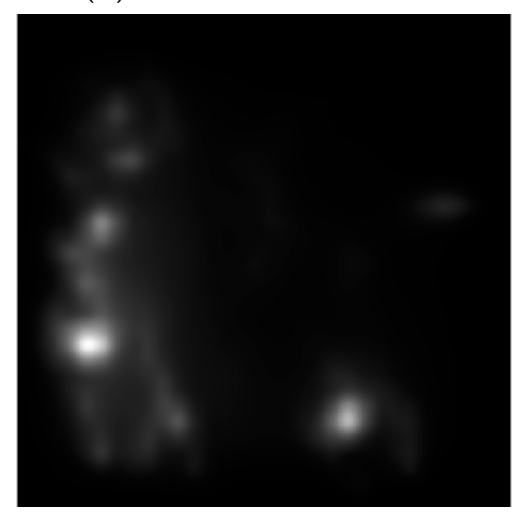

(e) result of RP method

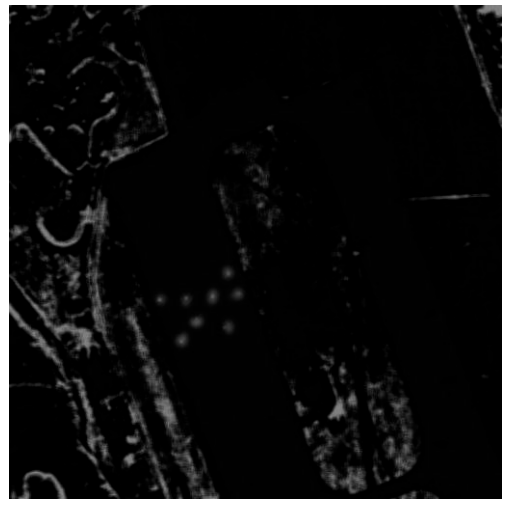

(c) result of SoP method

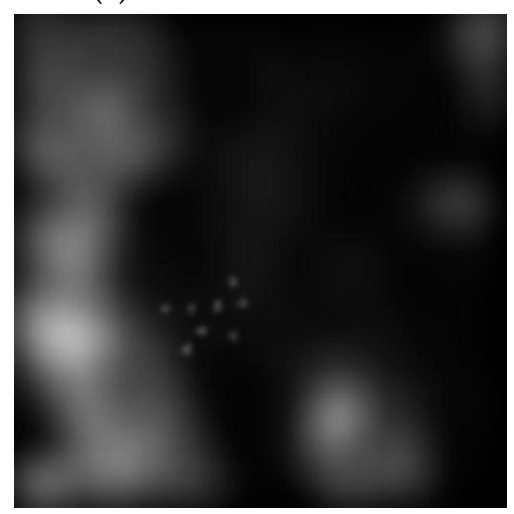

(f) result of proposed model

Figure 6. Saliency detection result of "Airport". 


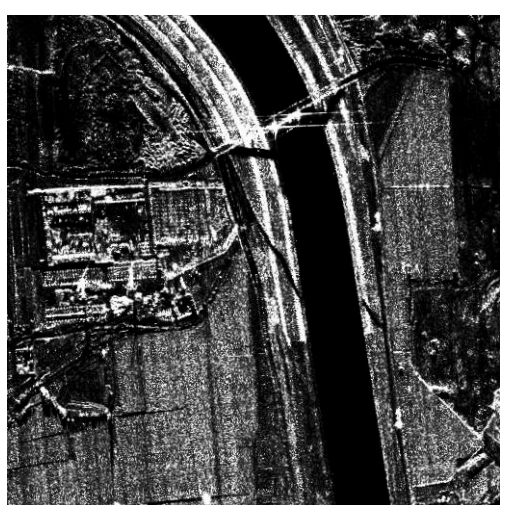

(a) original SAR image of "Bridge"

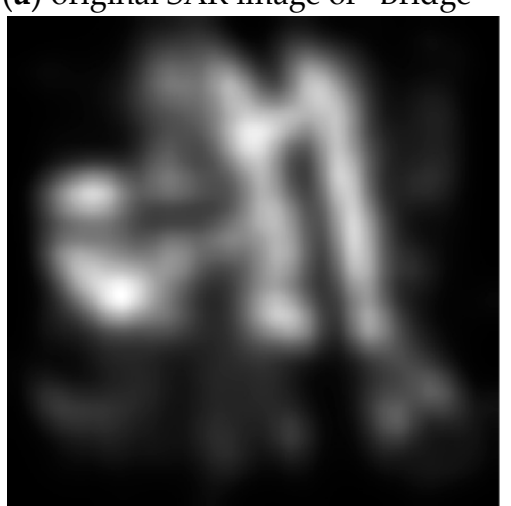

(d) result of PCT method

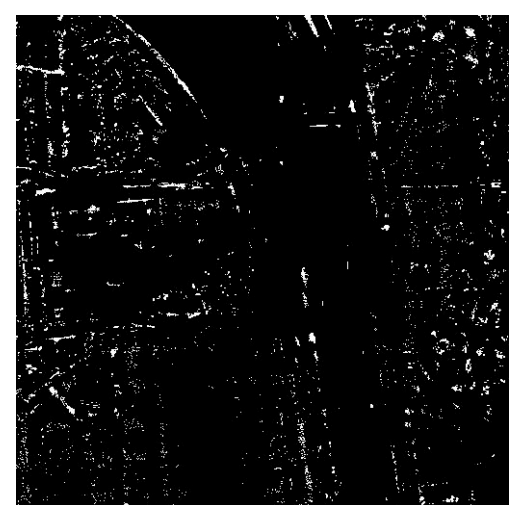

(b) result of CFAR method

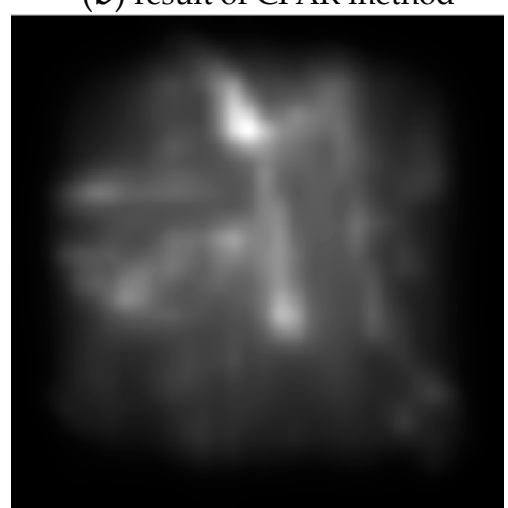

(e) result of RP method

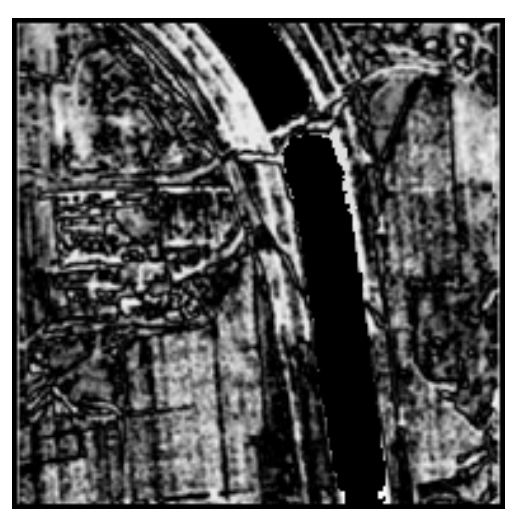

(c) result of SoP method

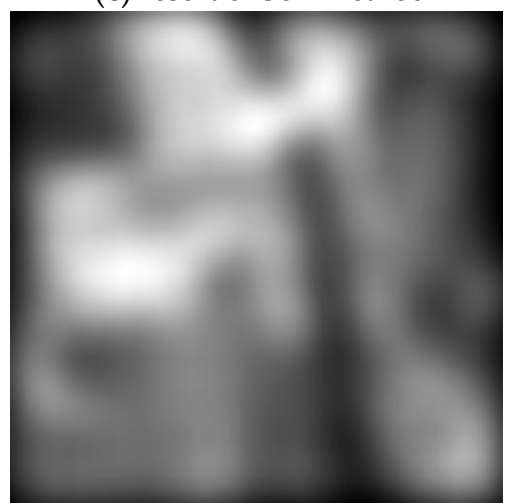

(f) result of proposed model

Figure 7. Saliency detection result of "Bridge".

For the case of Figure 5(a), main challenge for all the saliency detection methods is detecting the corner reflectors on the runway of the airport. All the methods that considered the local information work well in that regard, including CFAR, SoP and the proposed model. Among the three methods, the proposed MSD model can detect the salient area comprehensively and accurately. By contrast, the CFAR and SoP methods can only detect the local salient information such as edges or lines. The proposed method works better in terms of detection the salient areas of the background. This is exactly the strengths of proposed method that integrates the four typical features of SAR image. In contrary, the PCT and RP methods that only consider the global information fail to detect the detail information such as the corner reflectors and result in worse detecting performance than the proposed model.

The salient areas of Figure 5(c) include the bridge and its surrounding building areas, thus this case concentrates on testing the global saliency detecting ability of the five methods. The CFAR and SoP methods failed in this case, because both the two methods focus on the calculation of local intensity and the detecting results seems not comprehensive. In comparison, the other three saliency detectors, the PCT, RP, and the proposed methods have better detecting performance. Compared to the PCT and RP methods, the saliency map generated by the proposed method agrees better with our expectation.

In summary, the major advantage of the proposed MSD model would be both global and local saliency detection of the input image which is due to well integration of multiple features as well as the utility of the cross-feature information. Such advantage is more important for SAR images as it usually involves unknown size target or point target, and it is stressed by the above results.

As a quantitative evaluation, we compared the saliency detection results using the proposed method with respect to human-annotated "ground truth". For example, in Figure 3(a) and Figure $3(\mathrm{c})$, the corner reflectors and the vehicles on the bridge are first isolated by the red circles. Within each red circle, a threshold is applied to further label out pixels that are considered "target pixels". We define probability of detection $P_{d}$ at pixel level as the ratio between the number of detected pixels versus the number of annotated target pixels. On the other hand, the probability of false alarm $P_{f a}$ is 
defined as the ratio between the number of falsely detected pixels versus the total number of pixels detected.

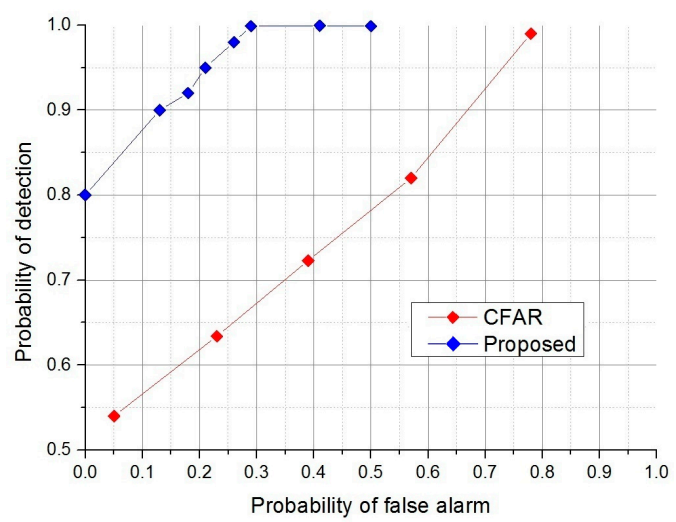

(a) ROC curves $P_{d}$ versus $P_{f a}$ of the "Airport"

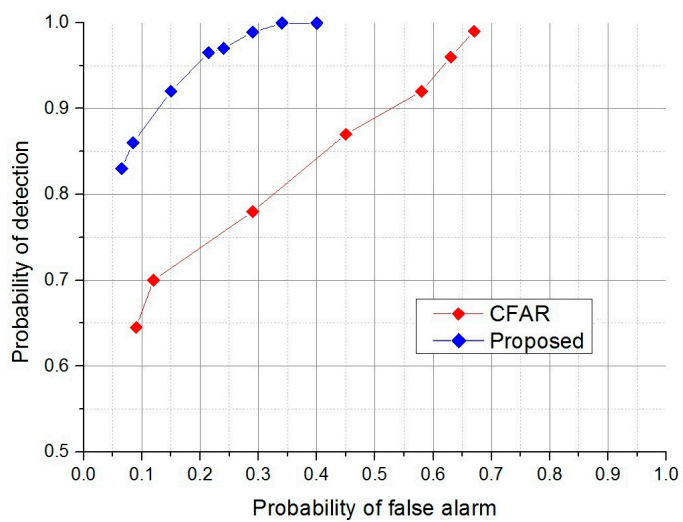

(b) ROC curves $P_{d}$ versus $P_{f a}$ of the "Bridge"

Figure 8. ROC curves of the saliency detection results.

Figure 8 plots the receiver operating characteristic (ROC) curves [27] of each case shown in Figure 6 and Figure 7. The proposed MSD saliency detector performs much better than standard CFAR-based detector. In Figure 8(a), at the false-alarm ratio $P_{f a}=21 \%$, the detection ratio $P_{d}$ of proposed method is 95\%, while for CFAR, it is about $P_{d}=64 \%$. In Figure $8(\mathrm{~b})$, the false-alarm ratio $P_{f a}$ of proposed method is $29 \%$ at the detection ratio $P_{d}=99 \%$, while it is $P_{f a}=78 \%$ for CFAR. We can draw the conclusions that the proposed method obtains much better performance than the classical CFAR method in the ability of saliency detection.

\subsection{Validation on $S A R$ and optical image fusion}

The proposed fusion method is compared with four fusion methods, including principal component analysis (PCA) [13], discrete wavelet transform (DWT) [15], non-sampled contourlet transform (NSCT) [16] and joint sparse representation (JSR) [17]. PCA is a spectral transformation that has been widely employed for pan-sharpening. DWT is developed by setting different fusion rules for combining the coefficients of low frequency and high frequency sub-bands of the two source image separately in discrete wavelet domain, but it sometimes produces Gibbs effect in some degree. NSCT is a multiple resolution analysis method which introduces has shift invariant property, and has been applied for remote sensing image fusion in recent years. JSR is a novel image fusion method, by which source image is represented with the common and innovation sparse coefficients based on joint sparse representation. The experiment results are shown in Figure 9 and Figure 10.

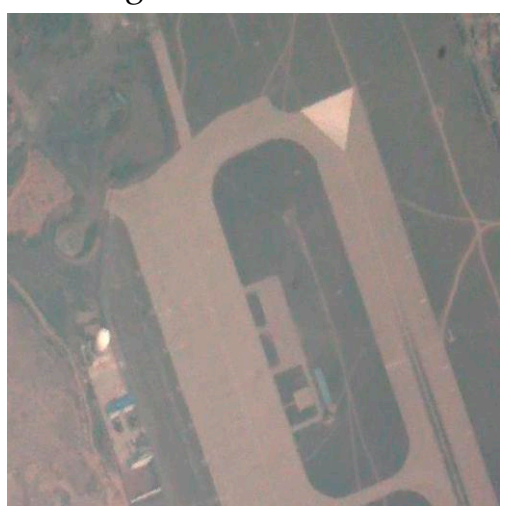

(a) original SAR image of "Airport"

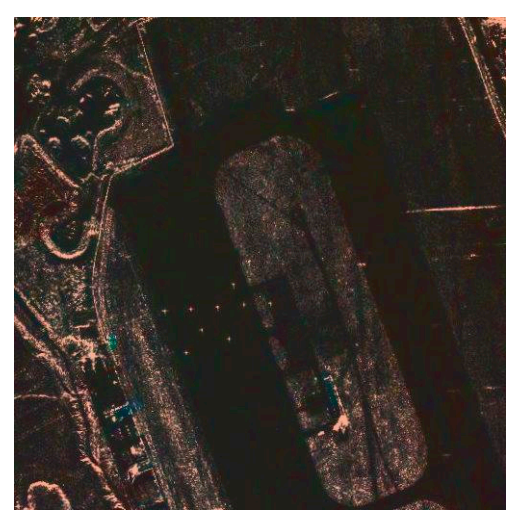

(b) result of PCA method

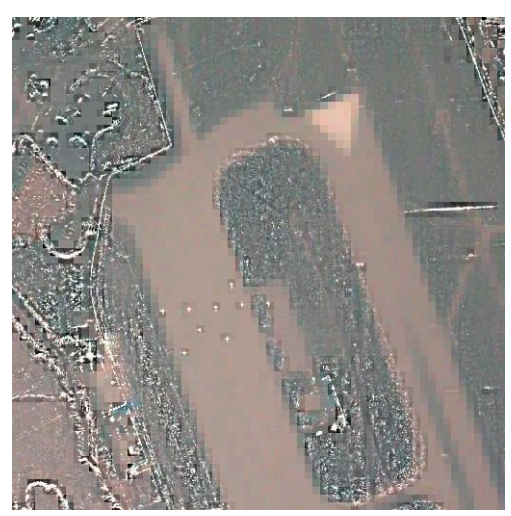

(c) result of DWT method 


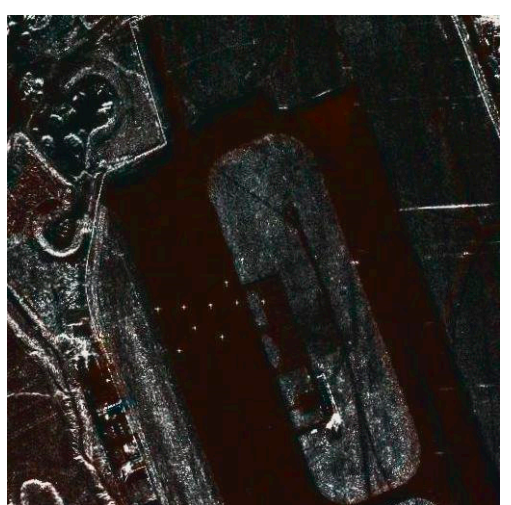

(d) result of NSCT method

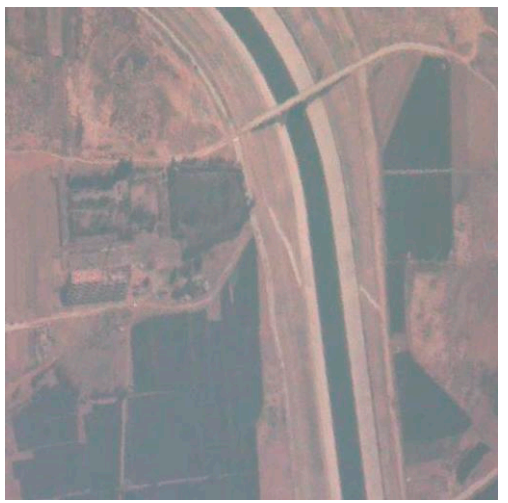

(a) original SAR image of "Bridge"

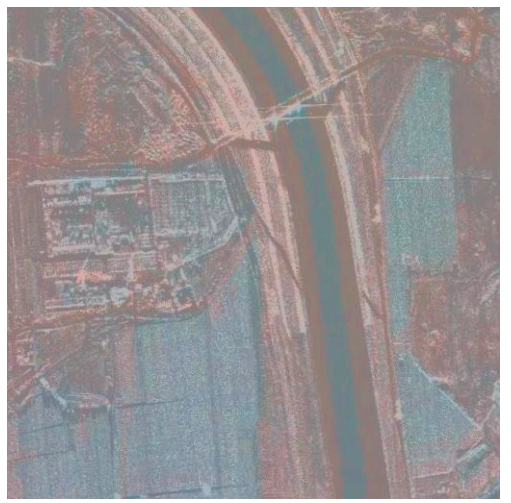

(d) result of NSCT method.

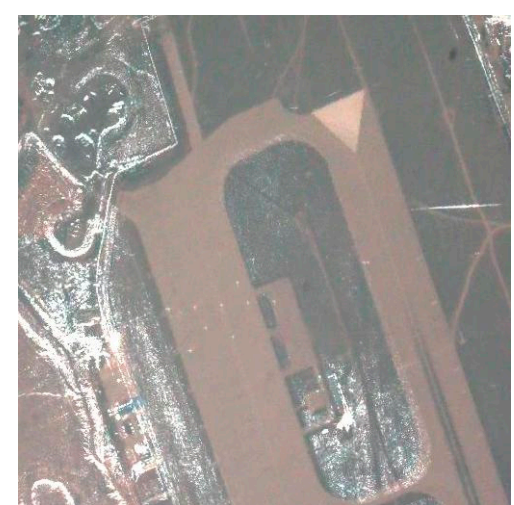

(e) result of JSR method

Figure 9. Fusion result of "Airport".

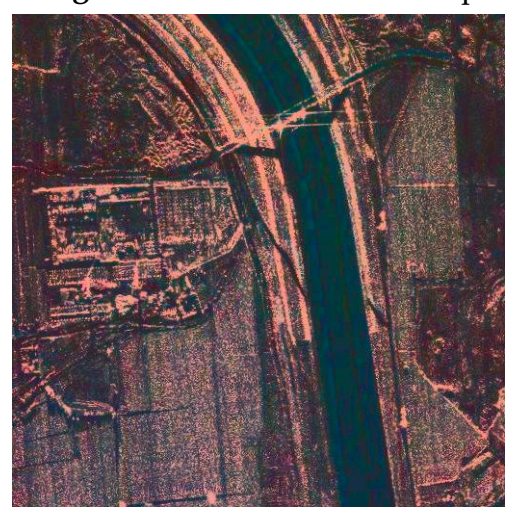

(b) result of PCA method

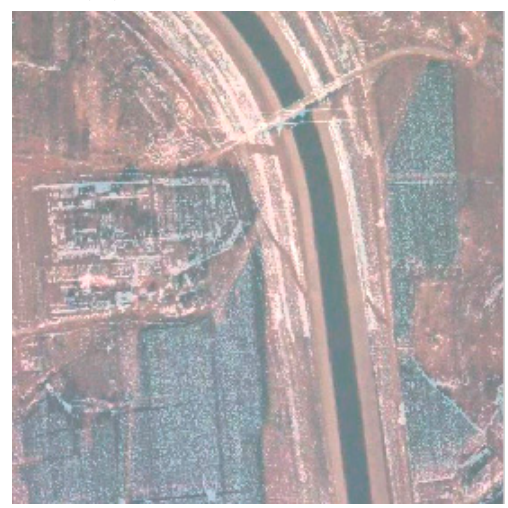

(e) result of JSR method

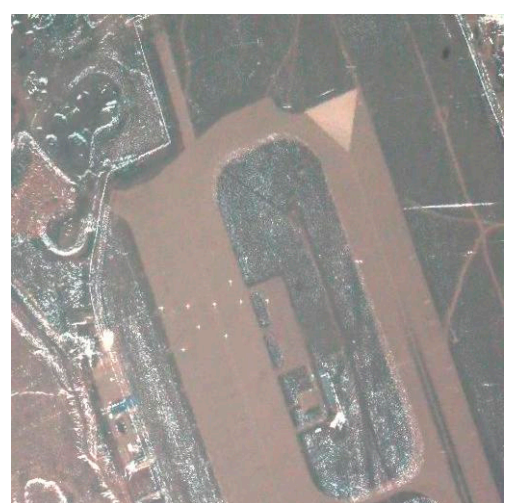

(f) result of proposed model

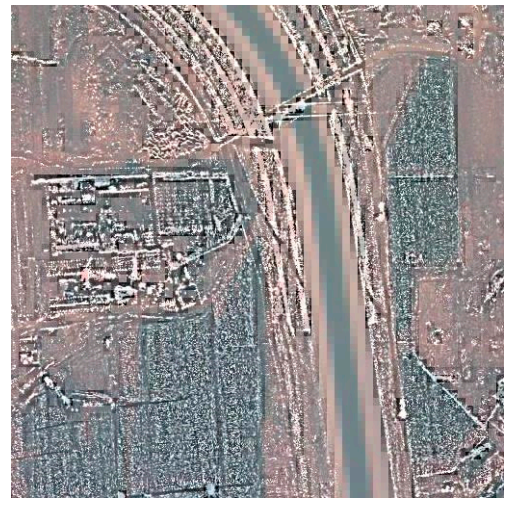

(c) result of DWT method

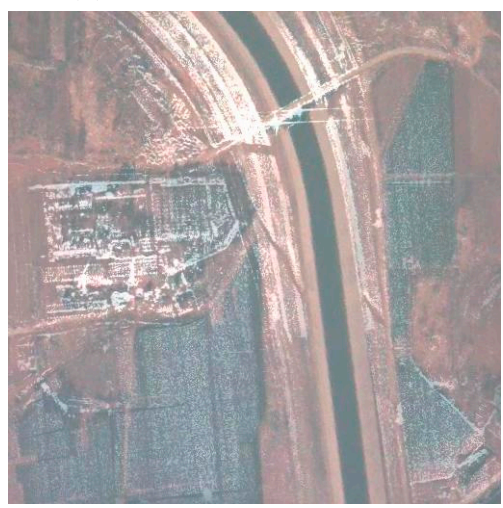

(f) result of proposed model

Figure 10. Fusion result of "Bridge".

As shown in Figure 9 and Figure 10, the spectral characteristics of the optical images have been distorted by PCA and NSCT methods, and this may cause the wrong interpretation of the target information. The fusion results by DWT method result in many speckle noises, though it can preserve the salient area in SAR image, especially the corner reflectors in Fig9(c). The results produced by JSR method contain detail spectrum information of the optical image and preserve the features of SAR image as much as possible, but it still introduces some speckle of SAR image. Comparing to the four fusion methods above, our proposed fusion method presents the best visual quality. Through visual observation, we can find that the fusion result by our method includes clear spatial information of the SAR image in the salient area with less speckle noise in non-salient area.

To evaluate the final fused image quality, we used four objective assessment metrics to evaluate the fusion results. The applied spectral estimators were the relative dimensionless global error in synthesis (ERGAS), the spectral angle mapper (SAM) and root mean square error (RMSE) [32,33]. The lower the ERGAS, SAM and RMSE are, the better the spectral similarity of the fused image to the original optical image. Meanwhile, the spatial correlation coefficient (sCC) [34] calculates the 
spatial similarity between the fused image and the SAR image, and the higher the value of sCC, the better the spatial similarity. The Quantitative results are reported in Table 1 and Table 2.

Table 1. Quantitative fusion results of "Airport".

\begin{tabular}{|c|c|c|c|c|c|c|}
\hline \multirow[t]{2}{*}{ Dataset } & \multirow[t]{2}{*}{ Metrics } & \multicolumn{5}{|c|}{ Methods } \\
\hline & & PCA & DWT & NSCT & JSR & Proposed \\
\hline \multirow{12}{*}{$\begin{array}{l}\text { Figure } 3 . \\
\text { (a)and (b) }\end{array}$} & ERGAS & 48.7851 & 3.9215 & 4.5212 & 3.3678 & 3.3426 \\
\hline & $\operatorname{SAM}\left({ }^{\circ}\right)$ & 5.1458 & 2.9743 & 4.8911 & 0.1524 & 0.1926 \\
\hline & RMSE & & & & & \\
\hline & $\mathbf{R}$ & 92.9372 & 26.7938 & 24.2016 & 15.3362 & 0.7312 \\
\hline & G & 91.0427 & 19.6666 & 24.2567 & 16.2526 & 0.5196 \\
\hline & B & 91.7178 & 21.1328 & 24.2563 & 19.7323 & 0.4239 \\
\hline & Avr & 91.8992 & 22.5310 & 24.2382 & 17.1070 & 0.5582 \\
\hline & sCC & & & & & \\
\hline & $\mathbf{R}$ & 0.01314 & 0.0711 & 0.02627 & 0.1282 & 0.1274 \\
\hline & G & 0.05618 & 0.0825 & 0.02816 & 0.0526 & 0.1612 \\
\hline & B & 0.01624 & 0.0989 & 0.03625 & 0.1356 & 0.1891 \\
\hline & Avr & 0.02852 & 0.0841 & 0.0302 & 0.1055 & 0.1592 \\
\hline
\end{tabular}

Table 2. Quantitative fusion results of "Bridge".

\begin{tabular}{|c|c|c|c|c|c|c|}
\hline \multirow[t]{2}{*}{ Dataset } & \multirow[t]{2}{*}{ Metrics } & \multicolumn{5}{|c|}{ Methods } \\
\hline & & PCA & DWT & NSCT & JSR & Proposed \\
\hline \multirow{12}{*}{$\begin{array}{l}\text { Figure } 3 . \\
\text { (a)and (b) }\end{array}$} & ERGAS & 7.6482 & 23.584 & 3.7182 & 3.8153 & 2.4451 \\
\hline & $\operatorname{SAM}\left({ }^{\circ}\right)$ & 6.9974 & 4.3086 & 0.24431 & 0.2209 & 0.3467 \\
\hline & RMSE & & & & & \\
\hline & $\mathbf{R}$ & 79.2344 & 43.5674 & 21.8714 & 18.634 & 14.551 \\
\hline & G & 75.2026 & 43.7683 & 21.2292 & 18.928 & 14.724 \\
\hline & B & 72.5521 & 43.7042 & 21.3347 & 18.327 & 14.462 \\
\hline & Avr & 75.663 & 43.68 & 21.4784 & 18.63 & 14.579 \\
\hline & $\mathrm{sCC}$ & & & & & \\
\hline & $\mathbf{R}$ & 0.06696 & 0.40236 & 0.3838 & 0.2313 & 0.1112 \\
\hline & G & 0.01879 & 0.22361 & 0.2072 & 0.1452 & 0.1314 \\
\hline & B & 0.06003 & 0.15325 & 0.2896 & 0.1951 & 0.1525 \\
\hline & Avr & 0.04859 & 0.25974 & 0.29353 & 0.1905 & 0.1317 \\
\hline
\end{tabular}

As shown in Table 1 and Table 2, the proposed method yields the best values of all of the spectral evaluation indices, except in the case of SAM values. This illustrates that the fused image produced by the proposed method has better maintained the spectral information of the optical image. From Figure 7 and Figure 8, we can also find that the proposed method does best in keeping the color information compared to the other four methods. We consider the low SAM values obtained for the results of JSR method to be due to the joint sparse decomposition and our method adopts the variational model. However, from the comprehensive comparison in both subjective and objective evaluation, it proves that our fusion method can preserve the spectral information from original color optical images in fusion results by including characteristics of the SAR data as much as possible. In addition, our algorithm yields a better sCC value than other methods. This may be due to the better injection of speckle pixels into the fused image. This allows our proposed method obtain the best similarity between the fused image and the SAR image. 
It can be seen from Table 1 and Table 2 that the proposed fusion method can provide the fusion result of better spectral and spatial qualities. Therefore, we can conclude that the proposed fusion method can maintain the feature of the salient region in the SAR images while preserving the color information of the optical image.

\section{Discussion}

Our work aims at giving a deep insight of the saliency information of SAR image and providing a possible application of the saliency map in the SAR and optical image fusion. Our results confirm that the proposed multitask saliency detection (MSD) model is accurate and effective for detecting the saliency of SAR image. Due to the efficient integrity of the multiple features of SAR image, the detecting ability of the MSD model is far better than the state-of-the-art methods. For the SAR and optical image fusion, based on the saliency map detected by MSD model, excellent fusion performance is obtained by the proposed variational fusion method.

Most existing saliency detection methods are local or global only, much less introducing the consideration of multiple features. Due to the characteristic of SAR images such as intensity concentration, the unknown size of target, full of speckle noise, etc, our method takes into account the typical features related to the areas of potential target before saliency detection. It makes the saliency maps generated by the MSD model more comprehensively covering the salient information of SAR image.

As mentioned in the introduction part, existing image fusion methods have not well used the salient information of the source images, especially in SAR and optical image fusion. The proposed fusion method can effectively integrate the saliency information into the fusion progress. The salient and non-salient areas can be preserved as much as possible in the fusion results and both subjective and objective evaluation verifies this point.

However, the computational complexity of the MSD model is relatively high, especially for real-time applications. It makes the MSD method more suitable for SAR image post-processing. One subject that remains to be explored is to find more features which can express the characteristic of SAR image for more specific applications, such as target recognition. In addition, the saliency map obtained by the MSD model is only tested on the image fusion experiment, other types of experiments adopted the saliency result need to be conducted.

\section{Conclusions}

A multitask saliency detection (MSD) model for SAR image is proposed which adopts the generic model of multitask sparsity pursuit (MTSP). Four features of synthetic aperture radar (SAR) image is extracted which include the intensity, orientation, uniqueness and global contrast. We establish the mathematical expressions and calculation method of the four features which can be the input of the MTSP. The saliency map of the SAR image can be obtained from the solution of the MTSP. Through subjective and objective comparison with several state-of-the-art saliency detecting methods, we can conclude that our proposed MSD model has better saliency detecting ability than the presenting methods.

We test the saliency detection result on SAR and optical image fusion application and proposed an image fusion method which guided by saliency maps. Due to introducing the salient information of the source images into the fusion procedure, the different features of the both the SAR and optical images can be maintained effectively. Accuracy assessment for real data shows a great potential of proposed fusion method. Future research can be directed towards to conducting more experiments of source data with various geographical features to adequately test the MSD model and the image fusion procedure. To well exploit the target information of the SAR image as the feature of the MSD model may also be worth investing effort.

Acknowledgments: This work was supported by National Natural Science Foundation of China (No. 61272348, 61572054). 
Author Contributions: C. L. performed the experiments and wrote the main part of the manuscript. Y. Q. wrote the abstract, introduction and conclusions. W. D. revised the manuscript.

Conflicts of Interest: The authors declare no conflict of interest.

\section{References}

1. Itti, L.; Koch, C.; Niebur, E. A model of saliency-based visual attention for rapid scene analysis. IEEE Trans. Pattern Anal. Mach. Intell. 1998, 20, 1254-1259.

2. Hou, X.; Zhang, L. Saliency detection: A spectral residual approach. In Proceedings of the 2007 IEEE Computer Society Conference on Computer Vision and Pattern Recognition (CVPR2007), Minneapolis, Minnesota, USA, 18-23 June 2007; pp. 1-8.

3. Guo, C.; Ma, Q.; Zhang, L. Spatio-temporal saliency detection using phase spectrum of quaternion Fourier transform. In Proceedings of the 2008 IEEE Computer Society Conference on Computer Vision and Pattern Recognition (CVPR2008), Anchorage, Alaska, USA, 24-26 June 2008; pp. 1-8.

4. Amoon, M.; Bozorgi, A.; Rezai-rad, G. New method for ship detection in synthetic aperture radar imagery based on the human visual attention system. Journal of Applied Remote Sensing 2013, 7, 071599-071599.

5. Bo, H.; Gu, H.Y; Sun, Q. Visual Attention Based SAR Image Small Target Detection. Journal of Computational Information Systems 2013, 9, 179-186.

6. Jäger, M.; Hellwich, O. Saliency and salient region detection in SAR polarimetry. In Proceedings of the IEEE International Geoscience and Remote Sensing Symposium (IGARSS2005), Seoul, Korea, 25-29 July 2005; pp. 2791-2794.

7. Zhang, X.H.; Meng, H.Y.; Ma Z.F.; Tian X.L. SAR image despeckling by combining saliency map and threshold selection. International Journal of Remote Sensing 2013, 34, 7854-7873.

8. Dou, F.; Diao, W.; Sun, X. Aircraft recognition in high resolution SAR images using saliency map and scattering structure features. In Proceedings of the 2016 IEEE International Geoscience and Remote Sensing Symposium (IGARSS2016), Beijing, China, 10-15 July 2016; pp. 1575-1578.

9. Wang, S.G.; Wang, M.; Yang, S.Y. New Hierarchical Saliency Filtering for Fast Ship Detection in High-Resolution SAR Images. IEEE Transactions on Geoscience E Remote Sensing 2017, 55, 351-362.

10. Song, T.; Yi, S. Fast and Accurate Target Detection Based on Multiscale Saliency and Active Contour Model for High-Resolution SAR Images. IEEE Transactions on Geoscience E Remote Sensing 2016, 54, 1-16.

11. Choi, M. A new intensity-hue-saturation fusion approach to image fusion with a tradeoff parameter. IEEE Transactions on Geoscience and Remote Sensing 2006, 44, 1672-1682.

12. Chien, C.L.; Tsai W.H. Image Fusion With No Gamut Problem by Improved Nonlinear IHS Transforms for Remote Sensing. IEEE Transactions on Geoscience and Remote Sensing 2014, 52, 651-663.

13. Shahdoosti, H.R.; Ghassemian, H. Combining the spectral PCA and spatial PCA fusion methods by an optimal filter. Information Fusion 2016, 27, 150-160.

14. Xu, H.; Wang Y.; Wu Y.; Qian Y. Infrared and multi-type images fusion algorithm based on contrast pyramid transform. Infrared Physics \& Technology 2016, 78, 133-146.

15. Khan, M.M.; Chanussot, J.; Condat, L.; Montavert, A. Indusion: Fusion of multispectral and panchromatic images using the induction scaling technique. IEEE Geoscience \& Remote Sensing Letters 2008, 3, 105-108.

16. Huang, H.D; Wang, B.; Zhang L.M. A new method for remote sensing image fusion based on nonsubsampled contourlet transform. Journal of Fudan University (Natural Science) 2008, 47, 124-128.

17. Yu, N.; Qiu, T.S.; Bi, F.; Wang, A. Image Features Extraction and Fusion Based on Joint Sparse Representation. IEEE Journal of selected topics in signal processing 2011, 5, 1074-1082.

18. Goferman, S.; Zelnik-Manor, L.; Tal, A. Context-aware saliency detection. IEEE Transactions on Pattern Analysis and Machine Intelligence 2012, 34, 1915-1926.

19. Itti, L.; Koch, C.; Niebur, E. A model of saliency-based visual attention for rapid scene analysis. IEEE Transactions on Pattern Analysis and Machine Intelligence 1998, 20, 1254-1259.

20. Liu, C.H.; Qi, Y.; Ding, W.R. Airborne SAR and optical image fusion based on IHS transform and joint non-negative sparse representation. In Proceedings of the 2016 IEEE International Geoscience and Remote Sensing Symposium (IGARSS2016), Beijing, China, 10-15 July 2016; pp. 1575-1578.

21. Yun, Y.; Choi, J.; Han, Y. An Area-Based Image Fusion Scheme for the Integration of SAR and Optical Satellite Imagery. IEEE Journal of Selected Topics in Applied Earth Observations \& Remote Sensing 2013, 6, 2212-2220. 
22. Lang, C.Y.; Liu, G.C.; Yu, J.; Yan, S.C. Saliency Detection by Multitask Sparsity Pursuit. IEEE Transactions on Image Processing 2012, 21, 1327-1338.

23. David, A. Computer Vision-A Modern Approach. Pearson Education, British,2002.

24. Socolinsky D.A. A variational approach to image fusion, Doctoral thesis, The Johns Hopkins University, USA, 2000.

25. Schölkopf, B; Platt, J; Hofmann, T. Graph-Based Visual Saliency. Advances in Neural Information Processing Systems 2006, 19, 545-552.

26. Piella, G. Image fusion for enhanced visualization: A variational approach. International Journal of Computer Vision 2009, 83, 1-11.

27. Hong, R.C.; Wu X.Q.; Wang, C. Multi-focus image fusion with salience preserving. Journal of University of Science E Technology of China 2008, 38, 1173-1179.

28. Gao, G. A parzen-window-kernel-based CFAR algorithm for ship detection in SAR images. IEEE Geoscience and Remote Sensing Letters 2011, 8, 557-561.

29. Huang, X.; Huang, P.; Dong, L.; Song, H. Saliency Detection Based on distance between patches in Polarimetric SAR Images. In Proceedings of the 2014 IEEE International Geoscience and Remote Sensing Symposium (IGARSS2014), Quebec, Canada, 13-18 July 2014; pp. 4572-4575.

30. Yu, Y.; Wang, B.; Zhang L. Hebbian-based neural networks for bottom-up visual attention and its applications to ship detection in SAR images. Neurocomputing 2011, 74, 2008-2017.

31. Wang, H.; Xu, F.; Chen, S. Saliency Detector for SAR Images Based on Pattern Recurrence. IEEE Journal of Selected Topics in Applied Earth Observations \& Remote Sensing 2016, 1-6.

32. Rahmani, S.; Strait, M.; Merkurjev, D.; Moeller, M.; Wittman, T. An adaptive IHS PAN-sharpening method. IEEE Geosci. IEEE Geoscience \& Remote Sensing Letters 2010, 7, 746-750.

33. Alparone, L.; Baronti, S.; Garzelli, A.; Nencini, F. A global quality measurement of PAN-sharpened multispectral imagery. IEEE Geoscience \& Remote Sensing Letters 2004, 1, 313-317.

34. Byun, Y.; Choi, J.; Han, Y. An Area-Based Image Fusion Scheme for the Integration of SAR and Optical Satellite Imagery. IEEE Journal of Selected Topics in Applied Earth Observations \& Remote Sensing 2013, 6, 2212-2220. 